\title{
THE AFRICAN GEOSPATIAL SCIENCES INSTITUTE (AGSI): A NEW APPROACH TO GEOSPATIAL TRAINING IN NORTH AFRICA
}

\author{
Stefan Oeldenberger and Karem Ben Khaled
}

3G-Tunisia, 2080 Ariana, Tunisia - (soelden, karem)@3g-consult.de

Commission VI, WG VI/4

KEY WORDS: AGSI, NGO, North Africa, Geospatial Qualification, Capacity Building, Certification, Cooperation

\begin{abstract}
:
The African Geospatial Sciences Institute (AGSI) is currently being established in Tunisia as a non-profit, non-governmental organization (NGO). Its objective is to accelerate the geospatial capacity development in North-Africa, providing the facilities for geospatial project and management training to regional government employees, university graduates, private individuals and companies. With typical course durations between one and six months, including part-time programs and long-term mentoring, its focus is on practical training, providing actual project execution experience. The AGSI will complement formal university education and will work closely with geospatial certification organizations and the geospatial industry.

In the context of closer cooperation between neighboring North Africa and the European Community, the AGSI will be embedded in a network of several participating European and African universities, e. g. the ITC, and international organizations, such as the ISPRS, the ICA and the OGC. Through a close cooperation with African organizations, such as the AARSE, the RCMRD and RECTAS, the network and exchange of ideas, experiences, technology and capabilities will be extended to Saharan and sub-Saharan Africa. A board of trustees will be steering the AGSI operations and will ensure that practical training concepts and contents are certifiable and can be applied within a credit system to graduate and post-graduate education at European and African universities.

The geospatial training activities of the AGSI are centered on a facility with approximately 30 part- and full-time general staff and lecturers in Tunis during the first year. The AGSI will operate a small aircraft with a medium-format aerial camera and compact LIDAR instrument for local, community-scale data capture. Surveying training, the photogrammetric processing of aerial images, GIS data capture and remote sensing training will be the main components of the practical training courses offered, to build geospatial capacity and ensure that AGSI graduates will have the appropriate skill-sets required for employment in the geospatial industry. Geospatial management courses and high-level seminars will be targeted at decision makers in government and industry to build awareness for geospatial applications and benefits. Online education will be developed together with international partners and internet-based activities will involve the public to familiarize them with geospatial data and its many applications.
\end{abstract}

\section{THE AGSI VISION}

"To provide best-in-region geospatial project training, geoinformation management and consulting services, contributing to the development of the North African region, flexibly and reliably through a network of trusted partnerships and close cooperation between North Africa and Europe".

\section{THE CURRENT MISSION}

Following the conceptual phase in 2011, the AGSI initiative is currently engaged in the setup of the supporting framework, before the AGSI can start with operations in 2013. During 2011 and 2012, several European Universities and also the ISPRS offered their support for the initiative. Supporting letters from the Tunisian government and important companies from the geospatial industry were obtained to further validate and to some extent modify the concept, ensuring no major obstacles remain ahead of the operations phase. However, initial financing is a concern, for which solutions must be found.

The setup of the AGSI society in Europe is in progress at this time. The society will work as a non-profit, independent NGO in Tunisia, where legal conditions now allow for the operations of international NGOs.

\section{BACKGROUND}

Following the momentous events of the 'Arab Spring' in North Africa, there is a real and opportune possibility to support the development of the private geospatial industry with numerous new employment opportunities. The people and governments of North Africa realize that their societies have to respond with urgency to the local and global challenges of the $21^{\text {st }}$ century. The most critical issues in the region are presented by:

1. Political instability;

2. Lack of security in the Saharan areas;

3. Expanding populations and pressure on public services, such as education and healthcare;

4. Very high youth unemployment;

5. The lack or wasteful use of natural resources;

6. Inefficient collection, treatment and recycling of solid and liquid waste and associated pollution;

7. Deficiencies in infrastructure management, current capacity, maintenance and new development;

8. The multitude of problems resulting from climate change, lack of water and progressing desertification.

Technology and especially the development of new infrastructure for renewable energy sources play a key role for 
the future of North Africa. Infrastructure projects must be supported by geospatial data and Geo-Information (GI) intelligence for all decision and life-cycle processes.

Geospatial data users in the region are facing a number of problems, due to the highly restrictive data policies of the past:

1. Geospatial data coverage at all mapping scales are mostly outdated and incomplete;

2. Lack of public awareness, public internet portals and e-government sites with geospatial information content;

3. Absence of high-resolution geospatial data sets Google Earth and Bing Maps represent the de facto reference standard even for government departments;

4. Lack of geospatial project and application knowledge, training and human resources development;

5. Insufficient funding for geospatial data collection, analysis, product generation and dissemination;

6. An underdeveloped private geospatial industry with insufficient capacity to meet actual requirements.

Developing nations such as India and China have been placing a substantial effort on the development of the geospatial information sector and are now benefiting from the availability of high quality data, supporting the development of their countries. Their success is a clear example that accurate, up-todate and high-resolution geospatial data can be collected and efficiently used by developing nations to accelerate sustained growth and improve associated management processes.

\section{THE AGSI IN TUNISIA}

The idea for the establishment of a geospatial training center in Tunisia's capital Tunis evolved in a series of discussions and meetings in 2011 among concerned geomatics professionals. It became clear that a new approach to rapid geospatial capacity building is required and in the aftermath of the 'Arab Spring' this concept has actually become feasible for the first time.

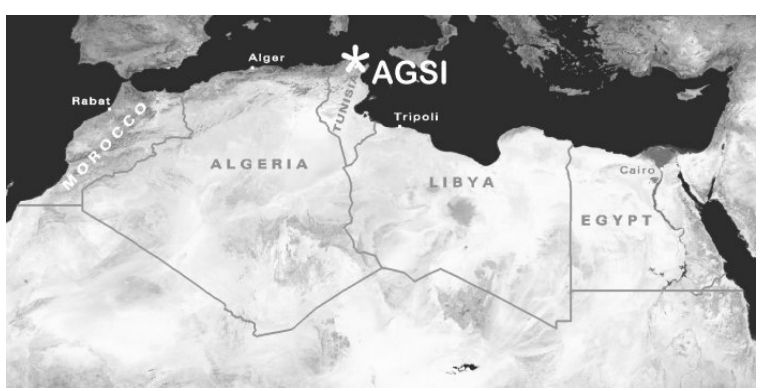

Fig. 1: AGSI Location in North Africa

The choice of Tunisia as the headquarter for the AGSI was influenced by its geographic location at the center of the southern Mediterranean, the easy access without visa restrictions to European and many African nationals and the long-term emphasis of Tunisian governments on education and the creation of a knowledge-based economy. All North African countries share Arabic as a common language and have traditionally very close cultural and personal ties across borders.

Given the multitude of common challenges and common interests, it is obvious that one of the main AGSI objectives must be to build and strengthen the geospatial cooperation in
Africa. The AGSI will connect to geospatial organizations, such as the African Association of Remote Sensing of the Environment (AARSE), incorporated in South Africa as a NGO, the Regional Centre for Mapping of Resources for Development (RCMRD) in Kenya and the Regional Centre for Training in Aerospace Surveys (RECTAS) in Nigeria.

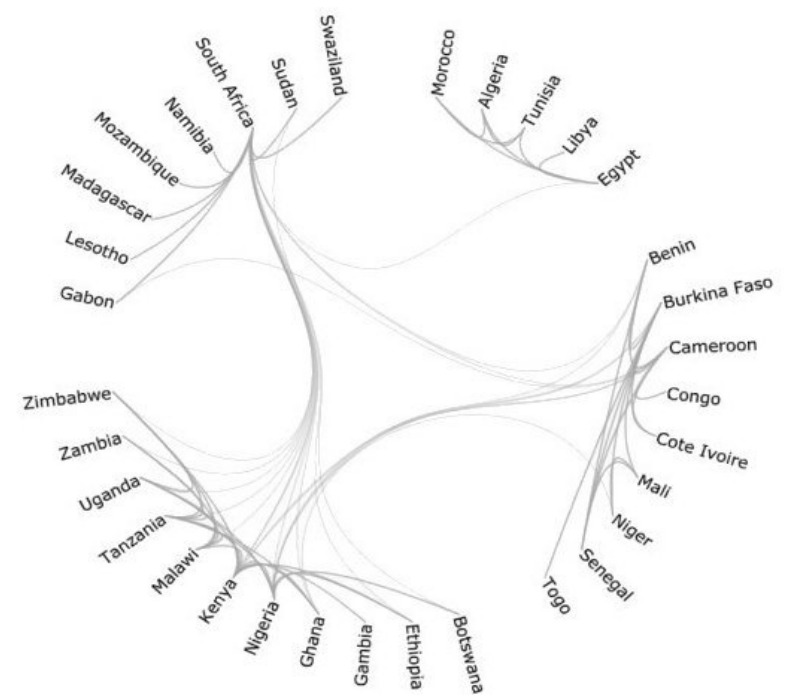

Fig. 2: African research clusters (Hook et al., 2010)

Based on partnerships of authors in the publication of research papers, Hook visualized the scientific links across Africa. It clearly shows the current, relatively isolated status of the North African countries in relation to the rest of the African continent.

\section{THE AGSI CONCEPT}

A non-profit, international NGO setup was selected to maintain a maximum of impartiality and independence for the AGSI, while providing financial and operational transparency to all partners and financial supporters. Only a strong, balanced network of partners, with active participation and support from international geospatial organizations and industry, universities, EU and local government, will ensure the success of the AGSI.

The AGSI board of trustees will ensure a constant flow of information between the partners, their delegates and the AGSI management. Decisions pertaining to regular AGSI operations will require a simple majority, while modifications to the AGSI statutes must be supported by a two-thirds majority.

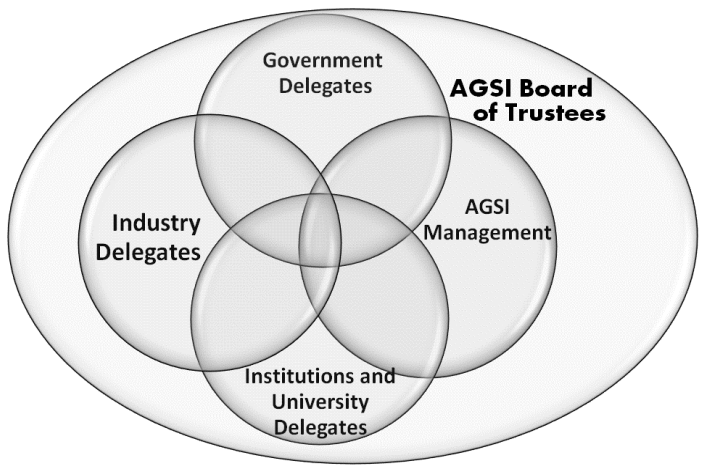

Fig. 3: AGSI Board of Trustees 
The combination of delegates from all stakeholders in a board of trustees provides a maximum of transparency and allows for efficient and structured communication, establishing majority positions and decisions for the AGSI operations.

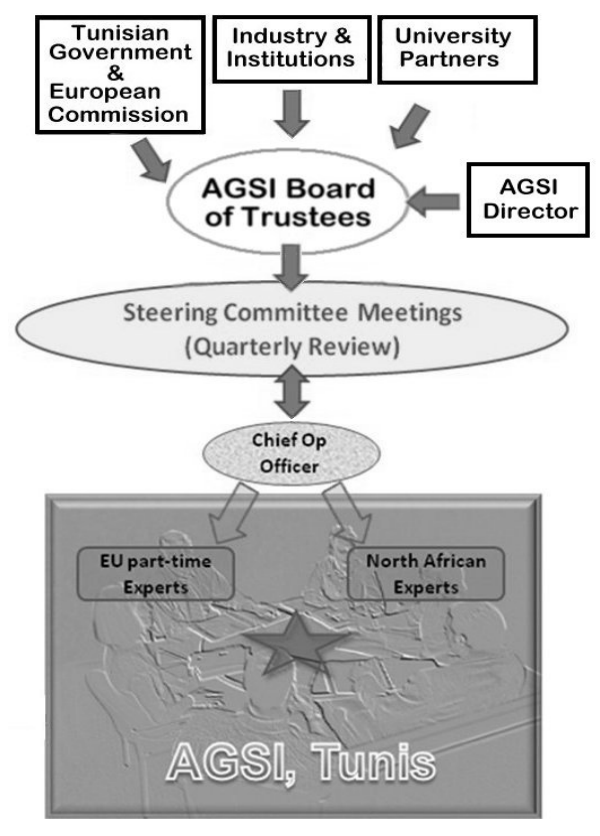

Fig. 4: The AGSI governing structure

The AGSI aims to efficiently fill the role as an intermediary between the more theoretical education at universities and industry requirements for personnel, where profound theoretical knowledge is backed up by practical skills and experience. It aims to be non-competitive and instead promote and support the objectives of the partners involved:

1. Compared to Universities, the AGSI does not offer long-term geospatial sciences programs with Bachelor, Masters or PhD degrees;

2. The AGSI training programs will be embedded in an international education credits and certification system, providing verifiable and transferable performance criteria for further education;

3. Compared to the geospatial industry, the AGSI training projects will be 'academic' in size, focusing on small areas, proving capabilities and encouraging growth and expansion to economic proportions;

4. Revenues will be invested into the development of training capabilities, business incubation activities and scholarships, both at the AGSI itself and abroad;

5. The AGSI will support certified graduates with office space to assist with the startup of their own companies.

As a geospatial technology and training center, the AGSI will draw and rely on international expertise, university lecturers and industry consultants to accompany the training programs. Local experts will play an important role from the beginning, especially if they have an international projects background.

In addition, consulting to local governments for the setup and management of geospatial projects and tenders will be a main activity of the AGSI and an important source of revenue.

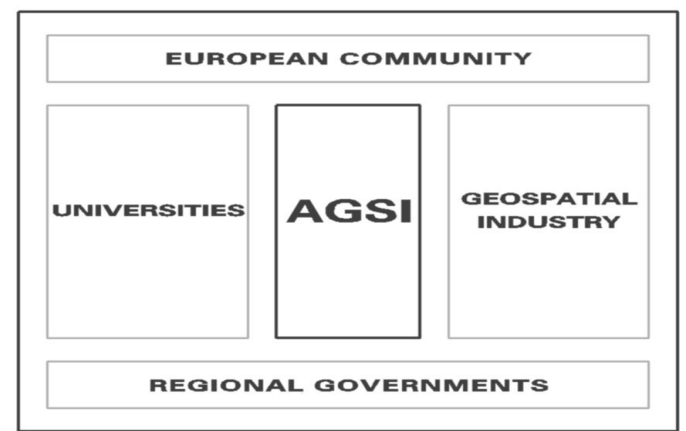

Fig. 5: AGSI schematic positioning

To be effective, geospatial training must be offered locally in North African countries, linked to liaison offices in Saharan and sub-Saharan countries. The AGSI regional headquarters will be established in Tunis in early 2013. A first branch in Tripoli, Libya will follow by late 2013. During the second phase in 2015, branches in Algiers, Cairo and Rabat will follow. The third phase, starting in 2017-2018, will see the opening of offices in adjacent Saharan countries. Liaison offices will have direct VSat connectivity to teleconference and podcast training.

The AGSI can not only fill a 'light-house' role for effective geospatial training and education, but also provide leadership in the networking of GI professionals, companies, universities, geomatics institutions and NGOs in North Africa. Functional networks are based on personal relations and shared involvement in joint programs. The involvement of international lecturers and joint participation in international programs form an important component for the creation of regional GI capacity.

The second component will be a necessary incubator function to be filled by the AGSI for small geospatial start-up companies founded by graduates. Temporary office space and the support by a network of professionals are definitive elements and further details of the incubator setup are under discussion.

\section{NSDI AND REGIONAL SDI SUPPORT}

The AGSI will be directly involved as a consulting organization and its support for the geospatial private sector in National Spatial Data Infrastructure (NSDI) programs and the regional African Spatial Data Infrastructure (ARSDI) initiative. As Molenaar and Mannaerts (2008) correctly state, geospatial data infrastructure (GDI) can be developed sustainably only through the active involvement of a strong private industry. Governments must have a regulatory role and provide an adequate GI infrastructure "but the growth of GDIs is possible only through public-private interactions". They also observe that finally it is the governments that benefit from better management of the environment and resources and that a competent GDI is indispensable for good governance at local, regional and international cooperation levels.

The deficiencies in the current status of SDI in North Africa can be explained largely to the absence of a mature geospatial industry. This is the result of historically restrictive data policies, decades of insufficient funding, technological isolation and lack of execution capacity for geospatial programs.

The European INSPIRE directive (EU 2007) for a common SDI in the European Union provides a ready basis for adaptation to 
North African requirements. For the first time, this would create comparable geospatial standards for data acquisition, sharing and dissemination across the region and geospatially connect North Africa with Europe. Regional road, railroad and integrated power grid projects are infrastructure examples which will directly benefit from the availability of national and regional SDI data. The considerable cost savings related to increased efficiencies during planning, maintenance and implementation of projects will by far offset the cost for national and regional SDI efforts.

\section{THE AGSI TRAINING AND EDUCATION SETUP}

The AGSI will pursue an educational and training approach that addresses four principal target groups to benefit from geospatial training, education and awareness:

1. The 'doers', i. e. the personnel directly involved in the technical execution of geospatial projects;

2. The geospatial project management staff, i. e. mid-level management in public administration and the geospatial industry;

3. The higher-level decision makers in government and the general industry to be informed about applications and benefits from geospatially-enabled technologies;

4. The wider public through online content and basic GI education at the level of secondary schools.

\subsection{Geospatial practical skills training}

For the 'doer' target group, the AGSI will deliver short (one to four weeks) and medium duration (one to six months) hands-on training courses, including evening classes, serving an approximate total of 100 participants during the first year. In the context of the three levels of geospatial professionalism and training defined by Ikhuoria (1999), the AGSI addresses the 'technical' skills level to build technical execution capacity.

Principal Geomatics courses at the technical skills level will be:
1. Surveying;
2. Photogrammetry;
3. Remote Sensing;
4. Digital Cartography;
5. Geographic Information Systems (GIS/LIS);
6. 3D terrain and urban areas modeling;
7. Mobile Mapping Systems;
8. Navigation data acquisition and processing;
9. Internet-based GI applications;
10.Geospatial database design.

In cooperation with the Institute for Photogrammetry (IFP) at the University of Stuttgart, the Munich University of Applied Sciences (MUAS) and other European Universities, highly performing AGSI participants may be selected and financially supported by scholarships for university education. Further educational partnerships will be entered into during the AGSI setup. All geospatial training activities will focus on practical execution and participants will gain direct hands-on experience in surveying, GIS, remote sensing, and aerial imaging projects.

\subsection{Geospatial management education}

For the second, non-technical target group, the AGSI will offer geospatial management courses, corresponding to the 'technological' and 'professional' level defined by Ikhuoria (1999). This group includes mainly administration officials and management personnel from the developing geospatial industry.

The following geospatial management subjects will initially be covered by the AGSI:

1. Geospatial project management;

2. Human resources management;

3. SDI concepts and implementation;

4. Environmental impact assessment.

\subsection{Geospatial seminars}

The third component of AGSI education activities will address decision makers in administration and the general industry through seminars and associated site visits. The objective is to raise awareness for opportunities and challenges that can be addressed by the efficient use of geospatial technology.

The following seminars and excursions will be offered:

1. Geospatial applications and benefits;

2. Renewable energies infrastructure;

3. Climate change and desertification.

\subsection{Geospatial awareness activities}

The AGSI activities will also familiarize the wider public through media and internet presence with geospatial applications and engage this group through crowd-sourced and participative GI activities on the AGSI website.

Basic geospatial education must be offered at the secondary schools level to create awareness and interest in pupils from an early age on. At this level, the AGSI aims to support geospatial education indirectly through programs for teachers, and directly through specific GI campaigns, such as summer-school classes for schoolchildren. Geospatial content at schools is the objective of the Digital Earth initiative (http://www.digital-earth.eu/), an example that can be adapted to the North African region.

\subsection{Geospatial online education}

The utilization of internet-based geospatial education resources and tools is essential and the AGSI intends to work closely with geospatial education organizations, such as the University of Nottingham, which is hosting the very advanced ELOGeo program (http://elogeo.nottingham.ac.uk/). The EU Agency for Education, Audiovisual and Culture Executive Agency (EACEA) is responsible for EU programs related to life-long learning in the fields of education, culture and audiovisual content and provides examples that could be applied to North African requirements for GI education, if and when supported by the respective governments and their education policies.

To enable the access to external resources for education and training, the AGSI will utilize high-speed internet connectivity for podcasts and live video conferencing. Using the example of photogrammetric classroom content, Fritsch (2007) has provided detailed descriptions on the creation, processing, distribution and access to educational podcast material.

The US-based Urban and Regional Information Systems Association (URISA) provides a comprehensive suite of online 
GIS education courses through its 'URISAConnect' program (http://www.urisa.org/URISAConnect). URISA has opened the first branch outside North America in 2007 and has started in 2011 to establish a chapter in the United Arab Emirates. Given the high importance of GIS technology for the sustainable development of North Africa, the adoption of existing online content and workshop programs are a priority for the AGSI to become operationally effective within a short period of time.

\section{THE AGSI FACILITIES SETUP}

\subsection{Staffing and building facilities}

Within the first year of operations, the AGSI will expand to approximately 24 North African staff to handle the technical, administrative and educational tasks, complemented by six to eight, mostly part-time international lecturers. In parallel to the expected increase in student numbers, the team is forecast to increase by $25 \%$ to around 40 staff in the second year, including 14 international GI professionals and lecturers.

Around $1,000 \mathrm{~m}^{2}$ of total floor space are required for the first phase of the AGSI training headquarters in Tunis. The initial setup plan calls for three classrooms, two student preparation rooms, lab facilities for GIS, photogrammetry, remote sensing, digital cartography and mapping, in addition to a fully equipped library with geospatial reference literature and magazines.

\subsection{Geospatial hardware}

As a training center with a strong focus on the practical execution of geospatial projects, the AGSI will have a considerable requirement for hardware and associated software to capture and process complete geospatial workflows and create for instance digital online and cartographic content.

\subsubsection{Airborne equipment}

The AGSI concept is centered on a small, single-engine survey aircraft with a medium-format digital aerial camera, airborne GPS, an Inertial Measurement Unit (IMU) and a Flight Management System (FMS) to plan and execute aerial image flights. LIDAR data acquisition and point cloud processing plays an important role in the geospatial data acquisition especially for precise surface models and consequently the AGSI will also seek to acquire a compact airborne instrument.

While the operation of an aircraft adds complexity to the operations of a geospatial training center, it allows for the flexible, rapid and high-resolution acquisition of aerial data, serving a multitude of project applications. The miniaturization of aerial equipment permits the use of a small, single engine aircraft with relatively low acquisition and hourly operations cost. The aerial equipment can be made available for local projects to the university partners of the AGSI, promoting the close cooperation. For MSc and PhD programs, such an aircraft certainly enables many research and validation capabilities.

The introduction of Unmanned Aerial Systems (UAS) for the aerial imaging of small areas is a second, very cost-efficient method to teach principles and basics of photogrammetry. In Europe, the use of UAS is strictly regulated, much depending on the weight of such systems. In North Africa it is also difficult to use UAS technology, because of safety and security reasons, although this is expected to change in the near future. A winged
UAS and a Quadcopter for 3D object capture will be purchased when the necessary operation permissions can be obtained.

\subsubsection{Surveying equipment}

Practical skills related to surveying data collection is essential for GI projects, aerial and satellite data processing. The AGSI will acquire two Global Navigation Satellite Systems (GNSS) base-stations and Real-Time Kinematic (RTK) rovers. These will integrate with a standard or robotic TotalStation for geodetic control, cadastral and monitoring survey applications.

Field reference data acquisition with mobile spectrometers is essential for the interpretation of multi-spectral remote sensing data. A field spectrometer is on the hardware acquisitions list for ground-truth data sets, required for the remote sensing training.

Several hand-held GNSS/GIS data collection systems are to be acquired for mobile workforce training, providing job skills essential for urban assets and utilities management, for the construction industry, environmental impact assessment and for rural surveying programs. GNSS/GIS hand-held data collection devices are also required for the completion of aerial image projects with ground-collected feature and attribute information.

\subsubsection{Processing equipment}

Aside from regular office computer equipment a small network of servers for GIS, imaging, associated products and internet applications will support the AGSI operations. Stereo-enabled workstations will be required for photogrammetric workflows.

\subsection{Geospatial software}

For the academic community and developing nations OpenSource software plays an important role. Strong competition drives development, performance, capabilities and reduces cost for commercial software. The main AGSI focus is on practical job skills training to facilitate the employment of participants by the geospatial industry. This necessitates a balanced approach to the utilization of both Open-Source and commercial software. In either case, emphasis is placed on OGC-compliant data formats to stress the indispensable interoperability across different software platforms and the support of SDI initiatives.

It cannot be the objective of the AGSI to become a training center for any one particular vendor platform, but instead must utilize a variety of software and hardware to remain impartial and independent. This approach encourages the comparison of capabilities, strengths and weaknesses of individual solutions.

\section{AGSI TRAINING CERTIFICATION}

The local and regional success of the AGSI will rely on the close cooperation with local and international certification bodies. The guidance of the universities and international organizations, present on the board of trustees, will ensure that training courses meet international standards. It is essential that courses are meeting international quality expectations and training results are verified in standard examinations. Successful participants must be able to transfer education credits obtained to international higher-level education and vocational programs, if they desire to continue with formal education.

On the AGSI board, the industry members will provide support and direction regarding the continued technical and professional 
development (CPD) of the students and their successful integration into the geospatial industry and work force.

\subsection{ECTS educational credits system}

The European Credit Transfer and Accumulation System (ECTS) will be adopted to support a close geospatial training cooperation with the European Community. The AGSI will provide the necessary setup for participants to obtain credits recognized by European vocational and graduate programs.

The ECTS User's Guide (EU 2009) provides the details on the framework created by the European Commission. ECTS can effectively be used to 'design, describe and deliver' educational programs and qualifications that are comparable and transparent in content to facilitate recognition of equivalent qualifications across the European Union and promote student mobility.

Within the ECTS system, 60 ECTS credits are equivalent to a full academic year or 1,500 to 1,800 hours of learning. Consequently, one ECTS credit corresponds to an academic workload of 25 to 30 hours. The AGSI training courses and higher-level seminars will be designed to be certifiable under the ECTS system. However, there is no formal agreement yet between the EU and North African countries regarding the acceptance of comparable educational credits. The initial objective is consequently to have AGSI educational credits accepted by partnering universities and institutions and thereby support cross-border certification efforts.

\subsection{ASPRS certification}

The AGSI curriculum will also be designed in cooperation with the ISPRS and ASPRS to support ASPRS certifications. ASPRS certification is an internationally accepted and recognized standard for professional achievements. Evening classes in combination with short, intense training courses at the AGSI and continuous mentoring are suitable direct learning tools to support the full range of ASPRS certification programs.

\subsection{GISCI certification}

The GIS Certification Institute (GISCI) has implemented certification procedures which assess the competency levels of GIS Professionals (GISPs). The certification procedures are based on points awarded for contributions to the profession, such as publications, workshops, participation in conferences and community work. The URISA is one of the founding members of the GISCI, and their GIS workshop and online training programs tie in with the GIS certification by the GISCI. Similar to the support for ASPRS certification, the AGSI will assess the existing background and geomatics skills of enrolled participants. AGSI training courses will enable participants to successfully attend and complete GISCI certification programs.

\subsection{ICA certification}

The International Cartographic Association (ICA) has started its own CPD program and provides implementation guidelines to enable a larger group of geospatial professionals to attain ICA certification. Fairbairn (2010) points out that the motivation for CPD programs is due to rapidly evolving new technologies and consequently, previously acquired skills become outdated after relatively short periods of time. Consequently, to maintain professional competence levels, geomatics companies and individuals alike should engage in CPD processes. The CPD program offered by the ICA is specifically designed for countries without existing, formal CPD programs of their own and could be applied in North Africa.

According to Gartner (2012) the current strategic objectives of the ICA are directed at:

1. Increased efforts for capacity-building, particularly in developing countries and especially concentrating on human resources development;

2. Facilitating the exchange of experts between and within technologically advanced and developing nations and revive the ICA, Third World ${ }^{\text {ee }}$ development policy.

\subsection{GI Certification at the AGSI}

The language barrier is considered as the main obstacle to international geomatics certification in North Africa. Most international education and certification programs are based on the English language, which is not widely spoken at sufficient proficiency levels in the region. Arabic is the only common language in North Africa but as such is not a technical language. French is used in Morocco, Algeria and Tunisia as a technical, professional language. English is not widely used in Libya anymore, but is comparatively widespread in Egypt, reflected in the better international scientific connectivity of the country.

Today, the vast majority of scientific publications related to GI sciences are published in the English language which is consequently a prerequisite, basic skill to stay current in GI technology and the latest developments. To base the AGSI curriculum and certification exclusively on the English language is desirable in this context and ultimately an advantage for the participants, but also problematic, adding a major prerequisite and barrier to enroll in AGSI training courses.

\section{FINANCING}

The AGSI aims to be financially self-sufficient as a non-profit NGO. It would be contradictory to many of its objectives to setup the AGSI as a commercial entity, competing in projects with private industry. The AGSI will be initially dependent on the financial support of donors, supporting governments and/or the European Community. Especially during the first year of operations, start-up investments have to be made in training facilities and required geospatial hardware.

The salaries paid to the AGSI staff account for approx. $38 \%$ of the overall budget. Competitive salaries are considered a necessity to attract the required talent and fulfill the AGSI leadership role as a knowledge-based GI sciences institution.

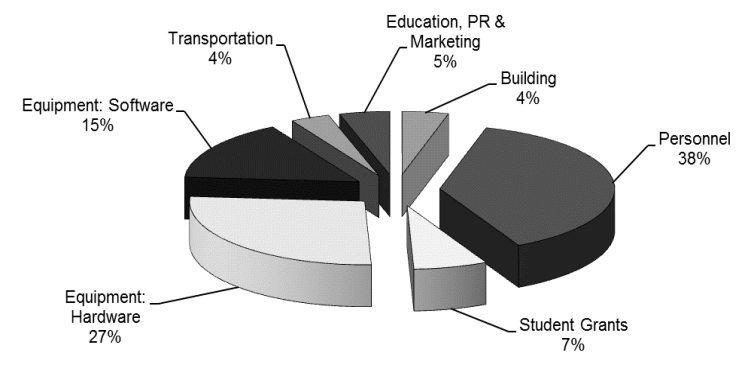

Fig. 6: $1^{\text {st }}$ year AGSI budget distribution 
During the first year of operations the financing of the AGSI will be based on four principal sources of income:

1. Funding by governments \& European Community;

2. Funding by industry and private donors;

3. Revenues from training courses;

4. Revenues from consulting services.

The North African countries have begun to recognize the benefits of a strong geospatial industry and have started to liberalize data acquisition and usage policies. Assuming this positive development will continue, the AGSI will meet its stated development goals and become financially self-sufficient within a period of three to four years.

\section{CHALlENGES}

North Africa in general faces many challenges, some of the most serious ones listed at the beginning of this paper. Specific challenges to the AGSI are related to a) the political understanding and will to invest in geospatial sciences and technology together with the required liberalization of geospatial data acquisition processes and data availability, and b) to the sufficient funding for the geospatial training programs.

The AGSI concept has enough flexibility to adapt to these two main challenges and still deliver and support quality geospatial education and certification, albeit at a lower than optimal pace and with slower growth and less diversity. Within the framework of this paper the following additional main challenges can only be listed briefly:

1. Passivity of the AGSI partner network;

2. Limited English language skills;

3. Weak local geospatial organizations;

4. Lack of professional identity;

5. Local governments slow to realize geospatial benefits;

6. Failure to reach public attention and build awareness.

As with all new concepts, the AGSI must be tried and tested and unforeseen challenges can be expected. With enough confidence, perseverance and broad-based professional support, the technical challenges can be overcome.

\section{CONCLUSION}

The African Geospatial Sciences Institute (AGSI) is in the process of being established with the support of the Tunisian government in Tunis. The concept for a local institute focusing on the delivery of practical geospatial training, high-level geospatial education and the creation of wider public awareness for applications and benefits of geospatial data appears sound and valid. The AGSI will be embedded in a network of universities, geomatics institutions, international organizations and the geospatial industry and will be beneficial to all parties involved. The North African countries will benefit from the creation of a competent geospatial industry which will be able to support and sustain the urgently required Spatial Data Infrastructure at the national and regional level.

The AGSI in Tunis is the first geospatial education facility to be established and serves as the regional center. The development of branch offices in North African is planned within the next three to five years to further strengthen the regional geospatial industry and build a network of geospatial professionals.
The positive civic development of North Africa, with a continued strengthening of its educational and institutional systems, can only take place if the international community and especially Europe as its northern neighbor will take an active role in the development of the region. The geospatial industry will act as a catalyst, enabling and accelerating the necessary maintenance and upgrade of the North African infrastructure, with the potential to create tens of thousands of jobs locally at all levels of professional qualification.

Today, North Africa and actually most European countries bordering the Mediterranean are suffering from high unemployment rates. The ongoing transition to renewable energy sources creates a new infrastructure and industry in Europe and North Africa which directly and decisively benefits our societies, our economies and the environment. Geospatial technologies and knowledge will strengthen the ties between North Africa and Europe and will provide a clear vision for a common future as neighbors across the Mediterranean Sea.

\section{ACKNOWLEDGEMENTS}

The authors of this paper would like to thank Prof. Ian Dowman and Prof. Dieter Fritsch for their early support and valuable comments regarding the development of the AGSI concept.

\section{REFERENCES}

EU 2007. "Directive 2007/2/EC of the European Parliament and of the Council of 14 March 2007 establishing an Infrastructure for Spatial Information in the European Community (INSPIRE) (14.03.2007). http://inspire.jrc.ec.europa.eu/index.cfm

EU 2009. ECTS User's Guide (06.02.2009), Office for Official Publications of the European Communities. Luxembourg http://ec.europa.eu/education/lifelonglearning/policy/doc/ects/guide_en.pdf

Fairbairn, D., D. Fraser, 2010. The role of international geomatics organisations in the promotion of continuing professional development (CPD). In: ISPRS Commission VI Mid-Term Symposium: Cross-Border Education for Global Geo-Information. 2010, ITC Enschede, The Netherlands: ISPRS.

Fritsch, D., 2007. Podcasting Photogrammetry - A Contribution to Life Long Learning. Photogrammetric Week '07, Ed. D. Fritsch, Wichmann, Heidelberg, pp. 335-343.

Gartner, G., 2012. Personal communication

Hook, D., J. Adams and C. King, 2010. Global Research Report Africa. Evidence, Thomson Reuters, Leeds, UK. http://researchanalytics.thomsonreuters.com/m/pdfs/globalresea rchreport-africa.pdf (Apr. 2010)

Ikhuoria, I., 1999. Professionalism and manpower issues in GIS in West Africa. Transactions in GIS, 3 (4), pp. 343-358.

Molenaar, M., C. Mannaerts, 2008. Capacity building networks for earth observation and Geo-ICT: a first step towards a virtual university. In: ISPRS 2008: Proceedings of the XXI Congress: The International Society for Photogrammetry and Remote Sensing, Beijing, China. Comm. VI, WG VI/3. pp. 93-96 